\title{
Acceptance or rejection of prophylactic medicine in patients with migraine: a cross-sectional study
}

\author{
Carianne MC Kol, Frans Dekker, Arie Knuistingh Neven, Willem JJ Assendelft and Jeanet W Blom
}

\begin{abstract}
Most patients with two or more migraine attacks per month do not use prophylactic medication. The aim of this study is to investigate how many patients use prophylaxis or would like to use them, and which aspects of migraine contribute to the choice to use prophylactic treatment. In a cross-sectional survey in three general practices, patients were selected who were diagnosed with migraine or had prescriptions for migraine medication. A questionnaire was sent to 283 patients and completed by 166 patients, of whom 15 were excluded. A total of 129 females and 22 males were included (median age 41 years). Most patients had two or more attacks per month (66.2\%). Fifty-five per cent of patients with two or more attacks per month wanted to use prophylaxis; only $8 \%$ actually used this treatment. To get more insight into the ideas for or against prophylactic use, qualitative research is indicated.

Keywords

headache; migraine; preventive therapy; prophylaxis; quality of life.
\end{abstract}

\section{INTRODUCTION}

Migraine is a common episodic headache disorder affecting about $6 \%$ of men and $15-18 \%$ of women in the general population, ${ }^{1,2}$ and is often associated with significant disability and reduced quality of life. ${ }^{3}$

Guidelines on migraine prophylaxis differ internationally, with prophylactic treatment recommended for patients with two attacks per month up to twice a week. According to guidance from the Dutch College of General Practitioners, patients with migraine attack frequency of more than two times per month should be offered prophylaxis. ${ }^{4}$ In the US, $50 \%$ of patients with migraine meet the criteria for use of prophylactic treatment, but only $5-12 \%$ actually use it. $^{3}$ In the Netherlands, $12 \%$ of all patients with migraine use prophylactic treatment. ${ }^{5}$

The first study aim was to investigate how many and which patients use prophylaxis, and how many patients would like to use this form of treatment. The second aim was to investigate how frequency, duration, severity, and impact of migraine attacks relate to the wish to use prophylactic treatment.

\section{METHOD}

\section{Study design and setting}

This was a cross-sectional survey conducted in three general practices with five GPs (approximately 10000 registered patients).

\section{Patient recruitment}

Patients aged 18 to 65 years diagnosed with migraine who were treated by their GP and recorded in the electronic patient register, were selected using the International Classification of Primary Care (ICPC). ${ }^{6}$ To identify patients with migraine who were not given a diagnosis according to the ICPC code, the database was searched for patients who had been prescribed migraine medication. Diagnostic codes were applied by the GP at any time from patient registration and after establishing the diagnosis.

\section{Data collection}

Data on patients' age, sex, number of visits, and the medication prescribed were collected from the 
electronic patient register. Data regarding frequency and duration of migraine attacks, migraine medication, and additional symptoms were selfreported using a mailed questionnaire.

The disease-specific quality of life of patients was measured with the Headache Impact Test (HIT-6), which is used to determine personal disease burden. ${ }^{7}$ The scores are categorised into four grades: representing 'minimal' (score of 49 or less), 'mild' (50-55 points), 'moderate' (56-59 points), or 'severe' (60 points or more). ${ }^{8}$

The questionnaire explored reasons for and against using prophylaxis, and whether patients preferred to ask their GP for information about prophylaxis, or preferred the GP to raise the issue. Explanations were presented in a simplified manner to make the information understandable to patients. The questionnaire asked the following:

'Consider a medicine which reduces your migraine symptoms by 50\%. This medicine would need to be taken every day. Ten per cent of patients have mild side-effects, such as dizziness, feeling cold, and fatigue. Would you be prepared to take this medicine?'

A reduction of more than $50 \%$ in disease burden is estimated to occur in over $50 \%$ of patients taking prophylactic therapy. ${ }^{9}$ For the remaining $50 \%$ of patients, the efficacy of prophylactic therapy is slightly less: for $\beta$ blockers an average reduction of $44 \%$ in migraine activity was shown. ${ }^{10}$

\section{Statistical analysis}

Associations between accepting prophylaxis and frequency, duration, and severity of migraine attacks, HIT-6 score, and consulting the GP were calculated using odds ratios with $95 \%$ confidence intervals. A logistic regression model was used to analyse associations between these variables and the willingness to consider prophylaxis independently from each other.

\section{RESULTS}

\section{Population description}

The questionnaire was sent to 283 patients, and completed by 166 patients (response rate $58.7 \%$ ). Thirteen patients stated in the questionnaire that they did not have migraine or had not experienced an attack for more than a year, and two patients did not answer the questions on prophylactic treatment. The study population consisted of 129 females (85.4\%) and 22 males (14.6\%). Median age was 41 years (interquartile range $=32-48$ years). Median HIT-6 score was 64 points (interquartile range =

\section{How this fits in}

Despite recommendations to offer prophylactic medication to patients with

frequent migraine attacks, studies indicate that only around $12 \%$ of patients

with migraine who meet criteria for prophylactic medication actually use this

form of treatment. It is unknown whether patients with migraine consulting their

GP are interested in using prophylaxis. This study shows that only $8 \%$ of

patients with two or more migraine attacks per month use prophylactic

medication. However, more than half of patients eligible for prophylaxis due to

their headache frequency are interested in using prophylactic medication and

should be actively informed by their GP.

60-68 points). Ten patients were already using prophylactic treatment.

\section{Response analysis}

Patients who completed the questionnaire visited their GP slightly more often in the previous year (56.6\% versus $46.2 \%, P=0.082$ ), and received medication for migraine more often than those who did not complete the questionnaire $(69.3 \%$ versus $55.6 \%, P=0.003$ ).

\section{Preference for prophylaxis}

Of the patients with two or more attacks per month, $7.9 \%$ already used prophylaxis. Most patients with migraine $(78.0 \%)$ with a low attack frequency (less than two times per month) did not want to consider using prophylaxis. However, the remaining $22.0 \%$ of patients with migraine with a low attack frequency also reported that they would be interested in trying prophylaxis. In the group of patients with two or more attacks each month, $55.4 \%$ reported that they would like to consider prophylactic treatment, whereas $80.0 \%$ of the patients with more than five attacks each month were interested.

Apart from the duration of migraine attack, the HIT-6 score, GP consultation during the year prior to this study, and use of migraine medication were associated with the willingness to consider using prophylaxis (Table 1). Most patients $(60.9 \%)$ felt sufficiently confident to ask their GPs about prophylaxis, rather than expecting the physician to initiate discussions. However, a substantial group of patients with migraine with an indication for prophylaxis expressed a wish to be informed by their GP about preventive treatment for migraine (39.1\%).

A logistic regression model showed that only frequency of migraine attacks was a strong independent determinant of desire for prophylaxis.

Patients who were against use of prophylaxis $(n=$ 84) gave the following reasons (more than one 
Table 1. Participant characteristics and preference for prophylactic treatment.

\begin{tabular}{|c|c|c|c|c|}
\hline Questionnaire & Total $(n=151)$ & Yes to prophylaxis & No to prophylaxis & Odds ratio $(95 \% \mathrm{Cl})$ \\
\hline \multicolumn{5}{|l|}{ Migraine frequency } \\
\hline$<2 \times$ per month & $50(33.1)$ & $11(22.0)$ & $39(78.0)$ & \\
\hline$\geq 2 \times$ per month & $101(66.2)$ & $56(55.4)$ & $45(44.6)$ & 4.4 (2.0 to 9.6$)$ \\
\hline$<5 \times$ per month & $111(73.5)$ & $35(31.5)$ & $76(68.5)$ & \\
\hline$\geq 5 \times$ per month & $40(26.5)$ & $32(80.0)$ & $8(20.0)$ & 8.7 (3.6 to 20.8$)$ \\
\hline \multicolumn{5}{|l|}{ Migraine duration ${ }^{\mathrm{a}}$} \\
\hline$<24$ hours & $58(38.9)$ & $23(39.7)$ & $35(60.3)$ & \\
\hline$\geq 24$ hours & $91(61.1)$ & $43(47.3)$ & $48(52.7)$ & $1.4(0.7$ to 2.7$)$ \\
\hline$<36$ hours & $111(74.5)$ & $45(40.5)$ & $66(59.5)$ & \\
\hline$\geq 36$ hours & $38(25.5)$ & $21(55.3)$ & $17(44.7)$ & 1.8 (0.9 to 3.8$)$ \\
\hline \multicolumn{5}{|l|}{ Additional symptoms } \\
\hline Not sensitive to light/sound & $32(21.2)$ & $11(34.4)$ & $21(65.6)$ & \\
\hline Sensitive to light/sound & $119(78.8)$ & $56(47.1)$ & $63(52.9)$ & 1.7 (0.8 to 3.8$)$ \\
\hline Nausea/vomiting absent & $41(27.2)$ & $16(39.0)$ & $25(61.0)$ & \\
\hline Nausea/vomiting & $110(72.8)$ & $51(46.4)$ & $59(53.6)$ & 1.4 (0.7 to 2.8$)$ \\
\hline Other & $49(32.5)$ & $28(57.1)$ & $21(42.9)$ & \\
\hline \multicolumn{5}{|l|}{ HIT-6 score ${ }^{b}$} \\
\hline$<60$ & $31(20.7)$ & $9(29.0)$ & $22(71.0)$ & \\
\hline$\geq 60$ & $119(79.3)$ & $58(48.7)$ & $61(51.3)$ & $2.3(1.0$ to 5.5$)$ \\
\hline \multicolumn{5}{|l|}{ Electronic patient register } \\
\hline \multicolumn{5}{|l|}{ Medication } \\
\hline No triptan use & $72(47.7)$ & $21(29.2)$ & $51(70.8)$ & \\
\hline Triptan use & 79 (52.3) & $46(58.2)$ & $33(41.8)$ & 3.4 (1.7 to 6.7$)$ \\
\hline No medication & $41(27.2)$ & $11(26.8)$ & $30(73.2)$ & \\
\hline \multicolumn{5}{|l|}{ Analgesic use } \\
\hline (excluding triptan use) & $31(20.5)$ & $10(32.3)$ & $21(67.7)$ & 1.3 (0.5 to 3.6$)$ \\
\hline \multicolumn{5}{|l|}{ Consultation frequency } \\
\hline Never & $86(57.0)$ & $29(33.7)$ & $57(66.3)$ & \\
\hline$\geq 1$ in the previous year & $165(43.0)$ & $38(58.5)$ & $27(41.5)$ & 2.8 (1.4 to 5.4$)$ \\
\hline
\end{tabular}

${ }^{\mathrm{a}}$ Two participants did not fill in this question. ${ }^{\mathrm{b}}$ One participant did not fill in this question.

answer was possible): fear of side-effects (38.1\%), experiencing minimal attacks (44.0\%), feeling as if by using daily medication they had a chronic disease (23.8\%), and 'other' (31.0\%).

\section{DISCUSSION}

\section{Summary of main findings}

In this study, most patients with migraine attacks of five or more per month, and about half of patients with two or more attacks per month, would like to consider prophylaxis. Even in the group of patients with fewer than two attacks per month, one in five would like to consider prophylactic treatment. Most patients felt sufficiently confident to approach their GP themselves for prophylaxis. However, a substantial subgroup preferred an active approach by their physician. This finding is confirmed in a British study which reports that patients often do not consult their GP for their headache symptoms but still would like more help. ${ }^{11}$

\section{Strengths and limitations of the study}

A weakness of this study is that the question used to inquire about the wish for prophylaxis is rather theoretical and does not adequately address individual patient motivations and situations influencing their needs. However, the findings indicate that further qualitative research into the different motivations of patients and doctors is required.

Patients in this study visited their GPs slightly more often and were prescribed triptans more than those who did not fill in the questionnaire. It is likely that those completed the questionnaire had a higher disease burden than those who did not fill it in. This might have caused a slight overestimation of the percentage of patients with migraine in general practice who wish to use prophylaxis.

\section{Comparison with existing literature}

In a recent study in the UK it was found that patients who were referred to neurologists more often consulted their GP and had more concerns about their headache symptoms. ${ }^{12}$ The current study's finding that patients who had seen their GP in the previous year were more likely to report an interest in prophylactic therapy, could also be explained by an increased concern about their symptoms. 


\section{Implications for clinical practice}

The present findings suggest that physicians can play a more active role in optimising migraine therapy for their patients. Many patients with migraine experience disability and absence from work. ${ }^{13}$ Better management by GPs could reduce individual suffering and have considerable gains for society.

\section{Competing interests}

The authors have stated that there are none

\section{Acknowledgements}

We thank the practices and patients involved in this study

\section{Discuss this article}

Contribute and read comments about this article on the Discussion Forum: http://www.rcgp.org.uk/bjgp-discuss

\section{REFERENCES}

1. Lipton RB, Scher AI, Kolodner K, et al. Migraine in the United States: epidemiology and patterns of health care use. Neurology 2002; 58(6): 885-894.

2. Lipton RB, Bigal ME, Diamond M, et al. Migraine prevalence, disease burden, and the need for preventive therapy. Neurology 2007; 68(5): 343-349.

3. Diamond M, Dahlof C, Papadopoulos G, et al. Topiramate improves health-related quality of life when used to prevent migraine. Headache 2005; 45(8): 1023-1030.

4. Knuistingh NA, Bartelink MEL, De Jongh TOH, et al. Standard for headache of the Dutch College of General Practitioners. Huisarts Wet 2004; 47(9): 411-422.

5. Rahimtoola H, Buurma H, Tijssen CC, et al. Incidence and determinants of migraine prophylactic medication in the Netherlands. Eur J Clin Pharmacol 2002; 58(2): 149-155.

6. Lamberts $\mathrm{H}$, Wood M. International classification of primary care. Oxford: Oxford University Press, 1987.

7. Headache Impact Test (HIT). The Netherlands (Dutch version) 1.1. Qualitymetric, Inc. and GlaxoSmithKline Group of Companies.

www.headachetest.com/HIT6/PDFS/Dutch\%20Belgium\%20.pdf (accessed 10 Jan 2008).

8. Kosinski M, Bayliss MS, Bjorner JB, et al. A six-item short-form survey for measuring headache impact: the HIT-6. Qual Life Res 2003; 12(8): 963-974.

9. Bigal ME, Lipton RB. The preventive treatment of migraine. Neurologist 2006; 12(4): 204-213.

10. Holroyd KA, Penzien DB, Cordingley GE. Propranolol in the management of recurrent migraine: a meta-analytic review. Headache 1991; 31(5): 333-340.

11. Belam J, Harris G, Kernick D, et al. A qualitative study of migraine involving patient researchers. Br J Gen Pract 2005; 55(11): 87-93.

12. Ridsdale L, Clark LV, Dowson AJ, et al. How do patients referred to neurologists for headache differ from those managed in primary care? Br J Gen Pract 2007; 57(538): 388-395.

13. Rasmussen BK. Epidemiology of headache. Cephalalgia 2001; 21(7): 774-777. 